Research Paper

\title{
microRNA-25 targets PKC $\zeta$ and protects osteoblastic cells from dexamethasone via activating AMPK signaling
}

\author{
Jian-bo Fan ${ }^{1,2, *}$, Wei Liu ${ }^{1, *}$, Xin-hui Zhu ${ }^{1}$, Hong Yi $^{1}$, Sheng-yu Cui ${ }^{1}$, Jian-ning Zhao ${ }^{2}$, \\ Zhi-ming Cui ${ }^{1}$ \\ ${ }^{1}$ Department of Orthopaedics, The Second Affiliated Hospital of Nantong University, Nantong 226001, Jiangsu, PR China \\ ${ }^{2}$ Department of Orthopaedics, Jinling Hospital, Nanjing Medical University, Nanjing 210008, Jiangsu, PR China \\ "These authors have contributed equally to this work \\ Correspondence to: Zhi-ming Cui, email: zhimingcuiorth@163.com \\ Keywords: dexamethasone, osteoblastic cells, microRNA-25, PKC, AMP-activated protein kinase (AMPK) \\ Received: July 27, $2016 \quad$ Accepted: November 14, $2016 \quad$ Published: November 29, 2016
}

\section{ABSTRACT}

\begin{abstract}
AMP-activated protein kinase (AMPK) activation could protect osteoblasts from dexamethasone (Dex). This study aims to provoke AMPK activation via microRNA downregulation of its negative regulator protein kinase C $\zeta$ (PKC ). Results show that microRNA-25-5p (miR-25-5p) targets PKC 's 3' untranslated regions (UTRs). Forced-expression of miR-25 downregulated PKC $\zeta$ and activated AMPK in human osteoblastic cells (OB-6 and hFOB1.19 lines), which thereafter protected cells from Dex. Reversely, expression of antagomiR-25, the miR-25 inhibitor, upregulated

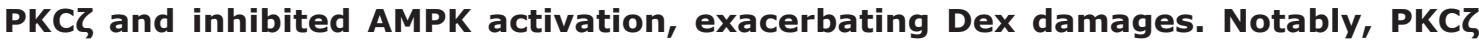
shRNA knockdown similarly activated AMPK and protected osteoblastic cells from Dex. AMPK activation was required for miR-25-induced osteoblastic cell protection. AMPKa shRNA or dominant negative mutation almost completely blocked miR-25induced cytoprotection against Dex. Further studies showed that miR-25 expression increased NADPH activity and suppressed Dex-induced oxidative stress in osteoblastic cells. Such effects by miR-25 were abolished with AMPKa knockdown or mutation. Significantly, miR-25-5p level was increased in patients' necrotic femoral head tissues, which was correlated with PKC downregulation and AMPK hyper-activation. These results suggest that miR-25-5p targets $\mathrm{PKC \zeta}$ and protects osteoblastic cells from Dex possibly via activating AMPK signaling.
\end{abstract}

\section{INTRODUCTION}

Long-term and/or excessive medication of dexamethasone (Dex) or other glucocorticoids (GC) will lead to different degrees of bone damages, including osteoporosis and osteonecrosis [1]. Osteoblast apoptosis was observed in the bones of GC-taking patients [1-3]. Dex could directly induce cytotoxic effect to osteoblasts, which may contribute to subsequent bone damages $[4,5]$. Dex was added directly to cultured osteoblasts/osteoblastic cells to mimic GC-induced bone damages [4, 6-9]. Groups including ours are studying the pathological mechanisms of GC-induced osteoblast damages, and developing possible intervention strategies [8-13].

AMP-activated protein kinase (AMPK) is a serine/ threonine protein kinase that is composed of a catalytic subunit $(\alpha)$ and two regulatory subunits ( $\beta$ and $\gamma)$ [14-16].
AMPK functions as a sensor of cellular energy status via phosphorylating its downstream targets, i.e. acetyl-CoA carboxylase (ACC) [14-16]. Recent studies suggested that AMPK and it-regulated signalings can also promote cell survival [17]. Activated AMPK inhibits oxidant stress via activating nicotinamide adenine dinucleotide phosphate (NADPH) and inhibiting ATP depletion [18]. Further, AMPK could also activate cytoprotective autophagy to inhibit cell apoptosis $[19,20]$.

Recent research has explored the potential function of AMPK in osteoblasts/osteoblastic cells. It was shown that AMPK activation by Compound 13, a novel AMPK activator [21], protected osteoblasts from Dex [7]. Similarly, Zhu et al., showed that A-769662, another AMPK activator, protected osteoblasts from hydrogen peroxide $\left(\mathrm{H}_{2} \mathrm{O}_{2}\right)$ [22]. Reversely, inhibition of AMPK, either genetically or pharmacologically, aggravated $\mathrm{H}_{2} \mathrm{O}_{2}-$ 
induced osteoblast cell death [23]. The results of these studies imply that AMPK activation exerts pro-survival functions in osteoblasts/osteoblastic cells.

Existing evidences suggested that protein kinase $\mathrm{C} \zeta(\mathrm{PKC} \zeta)$ may act as a negative regulator of AMPK [24]. Activated PKC $\zeta$ was shown to phosphorylate and inactivate LKB1, the AMPK kinase [24]. PKC $\zeta$ inactivation, knockdown or mutation resulted in LKB1 dephosphorylation and subsequent AMPK activation [24]. Based on the above information, it is proposed that $\mathrm{PKC} \zeta$ silence would activate AMPK and protect osteoblasts/ osteoblastic cells from Dex. Through searching the miRNA database (TargetScan v7.1), microRNA-25-5p ("miR-25-5p") is found to selectively targets PKC Importantly, we show that miR-25 downregulates $\mathrm{PKC} \zeta$ and protects osteoblastic cells from Dex possibly via activating AMPK signaling.

\section{RESULTS}

\section{microRNA-25 downregulates PKC $\zeta$ and activates AMPK signaling in human osteoblastic cells}

First, microRNA-25-5p ("miR-25-5p") indeed targets $\mathrm{PKC} \zeta$ 's 3' untranslated regions (UTRs, position 327-334) (Figure 1A). To test if miR-25 could downregulate $\mathrm{PKC} \zeta$ in osteoblasts, a pre-miR25-expressing construct was transfected it to OB-6 osteoblastic cells. Real-time PCR assay results in Figure 1B confirmed miR-25-5p over-expression in the stable OB-6 cells. miR-25 expression dramatically downregulated PKC $\zeta$ mRNA (Figure 1C) and protein (Figure 1D) expression in OB-6 cells. Significantly, AMPK activation, tested by $\mathrm{p}-\mathrm{AMPK}$ and $\mathrm{p}-\mathrm{ACC}$, was induced by miR-25 in OB-6 cells (Figure 1D). Similar results were also obtained in hFOB1.19 osteoblastic cells, where miR-25 expression (Figure 1E) downregulated PKC $\zeta$ (Figure 1F), and activated AMPK signaling (Figure $1 \mathrm{G})$. Notably, expression of miRNA control ("miR-C") had no such effects (Figure 1B-1G). The expression of microRNA-25-3p ("miR-25-3p") was also tested in these cells, and its level was not significantly increased following expression of pre-miR-25 (Supplementary Figure S1).

\section{microRNA-25 protects human osteoblastic cells from Dex}

Since AMPK activation would exert a pro-survival function in osteoblasts/osteoblastic cells [7, 22, 23], miR-25 expression should protect osteoblastic cells from Dex. As shown in Figure 2A, miR-25 expression indeed attenuated Dex-induced viability reduction in OB-6 cells.
Meanwhile, Dex-induced OB-6 cell apoptosis (Figure 2B) and cell death (Figure 2C) were also significantly inhibited by miR-25. Similarly in hFOB1.19 cells, miR-25 alleviated cell viability reduction (Figure 2D), apoptosis induction (Figure 2E) and cell death (Figure 2F) by Dex. On the other hand, expression of miR-C showed no such activity (Figure 2A-2F).

\section{antagomiR-25 exacerbates Dex damages in human osteoblastic cells}

To further confirm the role of miR-25 in osteoblastic cells, antagomiR-25, the miR-25 inhibitor, was introduced into OB-6 osteoblastic cells. Expression of antagomiR-25 did reduce miR-25-5p expression in OB-6 cells (Figure 3A, left). Reversely, PKC $\zeta$ expression was upregulated (Figure 3A right, and 3B). Consequently, AMPK activation, or p-AMPK/p-ACC, was decreased in antagomiR-25-expressing cells (Figure 3B). Further studies showed that antagomiR-25 exacerbated Dexinduced OB-6 cell damages, leading to enhanced viability reduction (Figure $3 \mathrm{C}$ ) and apoptosis activation (Figure 3D). Therefore, antagomiR-25 downregulates miR-25-5p and inhibits AMPK activation, which then potentiates Dex damages in human osteoblastic cells.

\section{PKC $\zeta$ knockdown activates AMPK and protects human osteoblastic cells from dex}

To test whether PKC $\zeta$ downregulation was the reason of AMPK activation in miR-25-expressing osteoblastic cells, shRNA strategy was applied to knockdown PKC $\zeta$ in OB-6 cells. Two non-overlapping PKC $\zeta$ shRNAs ("-1/-2") were applied, and each of them caused dramatic $\mathrm{PKC} \zeta$ downregulation (protein and mRNA) in OB-6 cells (Figure 4A and 4B). miR-25-5p level was unchanged with $\mathrm{PKC} \zeta$ knockdown (Figure 4A). Remarkably, PKC $\zeta$ shRNA induced significant AMPK activation (p-AMPK/p-ACC) in OB-6 cells (Figure 4B), showing similar phonotype of miR-25 over-expression (Figure 1). More importantly, OB-6 cells with PKC $\zeta$ silence were also protected from Dex, presenting with reduced viability reduction (Figure 4C) and apoptosis (Figure 4D).

miR-25 was then expressed in the PKC $\zeta$-silenced cells. qRT-PCR results confirmed miR-25-5p overexpression (Figure 4E) and $\mathrm{PKC} \zeta$ downregulation (Figure 4F) in these cells. Intriguingly, expression of miR-25 failed to further protect OB-6 cells from Dex in $\mathrm{PKC} \zeta$-silenced cells (Figure 4G and $4 \mathrm{H}$ ). In another words, miR-25 appeared in-effective against Dex when PKC $\zeta$ was depleted (Figure $4 \mathrm{G}$ and $4 \mathrm{H}$ ). These results imply that $\mathrm{PKC} \zeta$ might be the primary target of miR-25 in mediating its cytoprotective effect in osteoblastic cells. 


\section{AMPK activation is required for miR-25- induced cytoprotection in osteoblastic cells}

Thus, miR-25 expression activated AMPK and protected osteoblastic cells from Dex. The link between the two was then explored. Genetic strategies were utilized to block AMPK activation. AMPK $\alpha$ shRNA or dominant negative AMPK $\alpha$ ("dn-AMPK $\alpha$ ", T172A) was introduced to the miR-25-expressing OB-6 cells. Western blot results in Figure 5A demonstrated that

A.

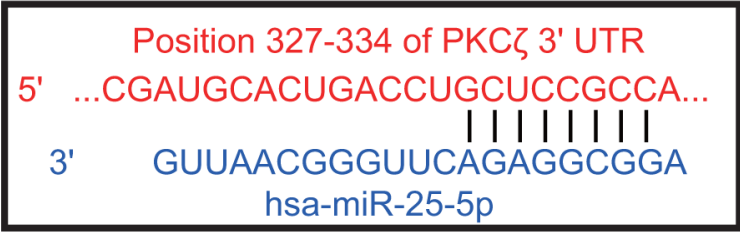

B.

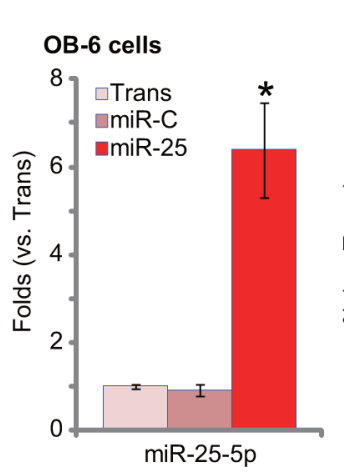

C.

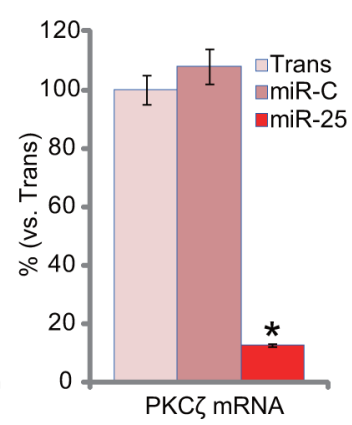

D.
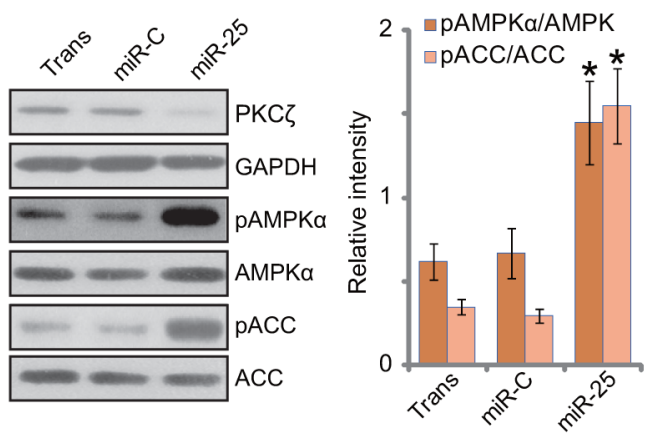

E. hFOB1.19 cells

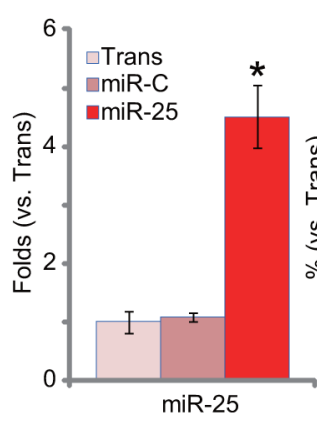

$\mathbf{F}$.

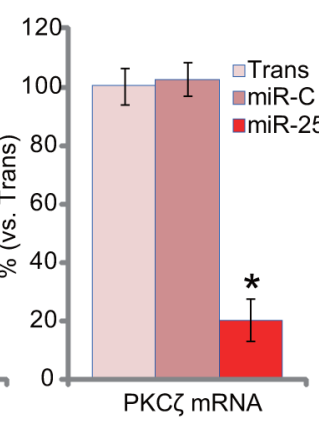

G.

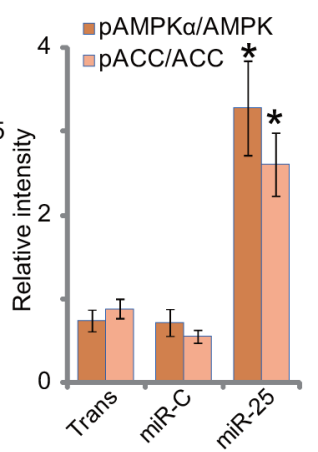

Figure 1: microRNA-25 downregulates PKC $\zeta$ and activates AMPK signaling in human osteoblastic cells. A. microRNA25-5p ("miR-25-5p") targets the 3' untranslated regions of human PKCל. Human osteoblastic OB-6 cells B-D. or hFOB1.19 cells E-G. were transfected with has-pre-miR-25 or the control microRNA ("miR-C"), and stable cells were established; Expressions of miR-25-5p (B and E) and PKC $\zeta$ mRNA (C and F) were tested by quantitative real-time PCR ("qRT-PCR") assay; Expression of listed proteins in these cells was tested by Western blot assay, and phosphorylations of AMPK $\alpha$ and ACC $\alpha$ were quantified (D and G). Experiments in this figure were repeated three times, and similar results were always obtained. "Trans" stands for transfection reagents only (B-G). * $p<0.05$ vs. group "miR-C" (B-G). 
A.

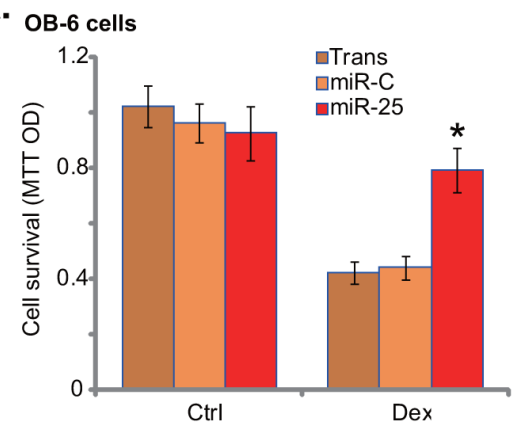

D.

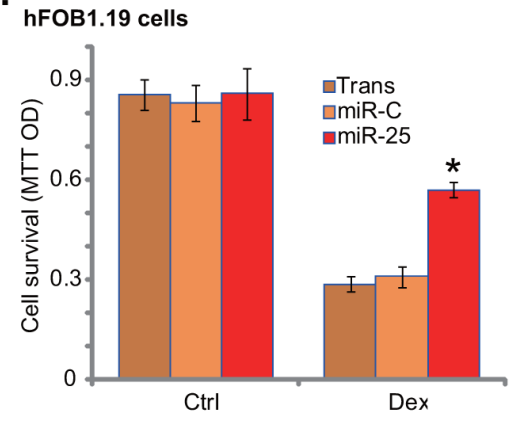

B.

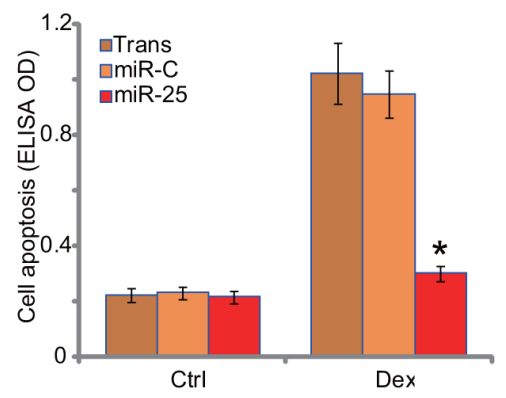

E.

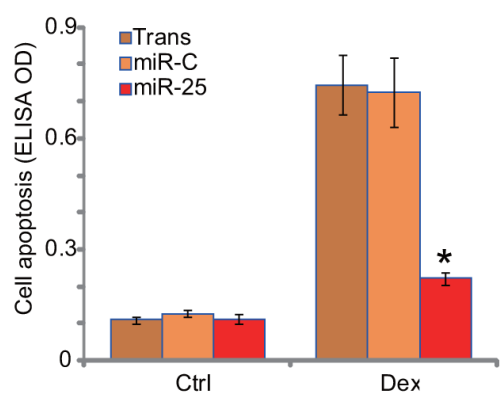

C.

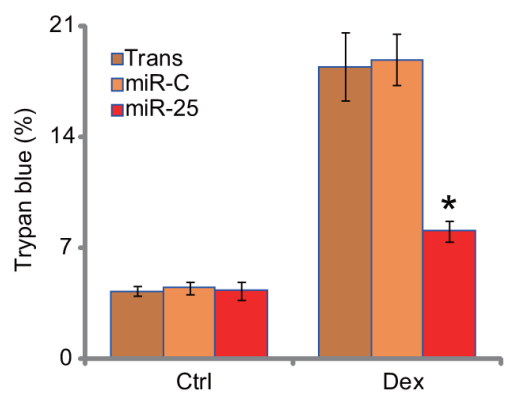

$\mathbf{F}$.

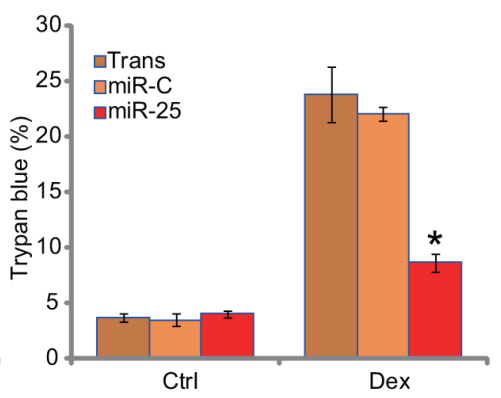

Figure 2: microRNA-25 protects human osteoblastic cells from Dex. Stable osteoblastic OB-6 cells A-C. or hFOB1.19 cells D-F. expressing microRNA-25 ("miR-25") or control microRNA ("miR-C") were treated with or without Dex $(1 \mu \mathrm{M})$ for 24 hours, cell viability (MTT assay, A and D), cell apoptosis (Histone DNA ELISA assay, B and E) and cell death (trypan blue assay, C and F) were tested. Experiments in this figure were repeated three times, and similar results were obtained. "Ctrl" stands for untreated control group. "Trans" stands for transfection reagents only. * $p<0.05$ vs. "miR-C" cells with Dex treatment.

A.

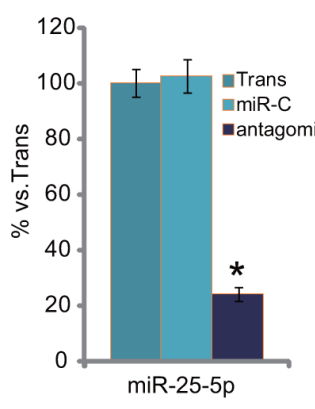

C.

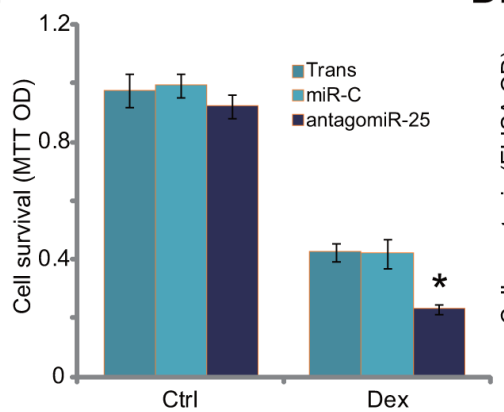

D.

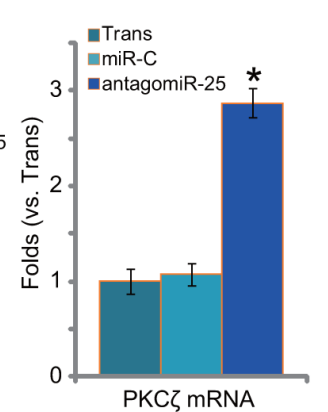

B.
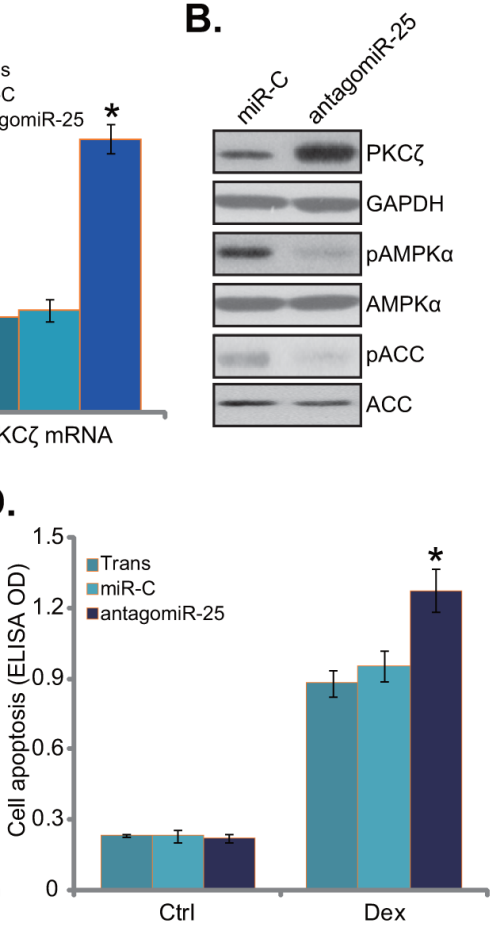

Figure 3: antagomiR-25 exacerbates Dex damages in human osteoblastic cells. Expressions of miR-25-5p (A, left), PKC $\zeta$ mRNA (A, right), and listed proteins B. in stable osteoblastic OB-6 cells with antagomiR-25 or non-sense control microRNA ("miR-C") were shown; Above cells were treated with or without Dex $(1 \mu \mathrm{M})$ for 24 hours, cell viability (MTT assay, C.) and apoptosis (Histone DNA ELISA assay, D.) were tested. Experiments in this figure were repeated three times, and similar results were obtained. "Ctrl" stands for untreated control group. "Trans" stands for transfection reagents only. * $p<0.05$ vs. "miR-C" cells. 
A.

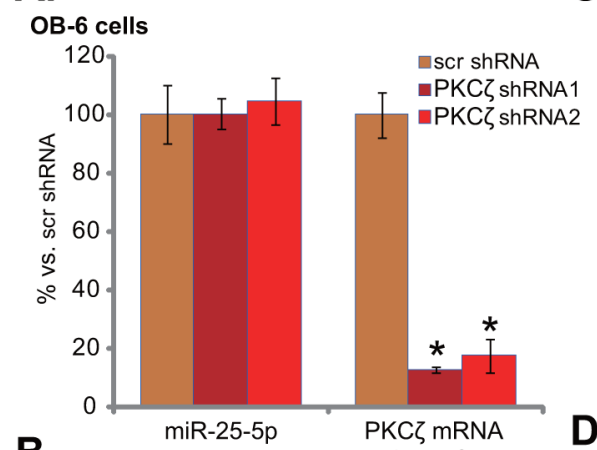

B.

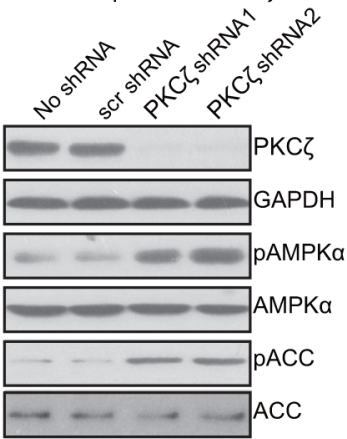

E.

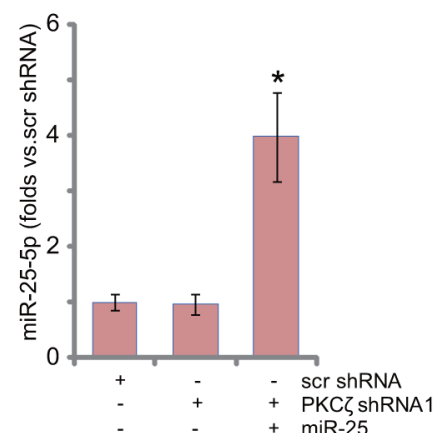

F.

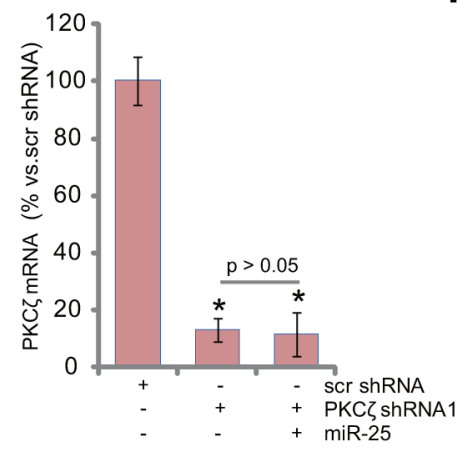

G.
C.
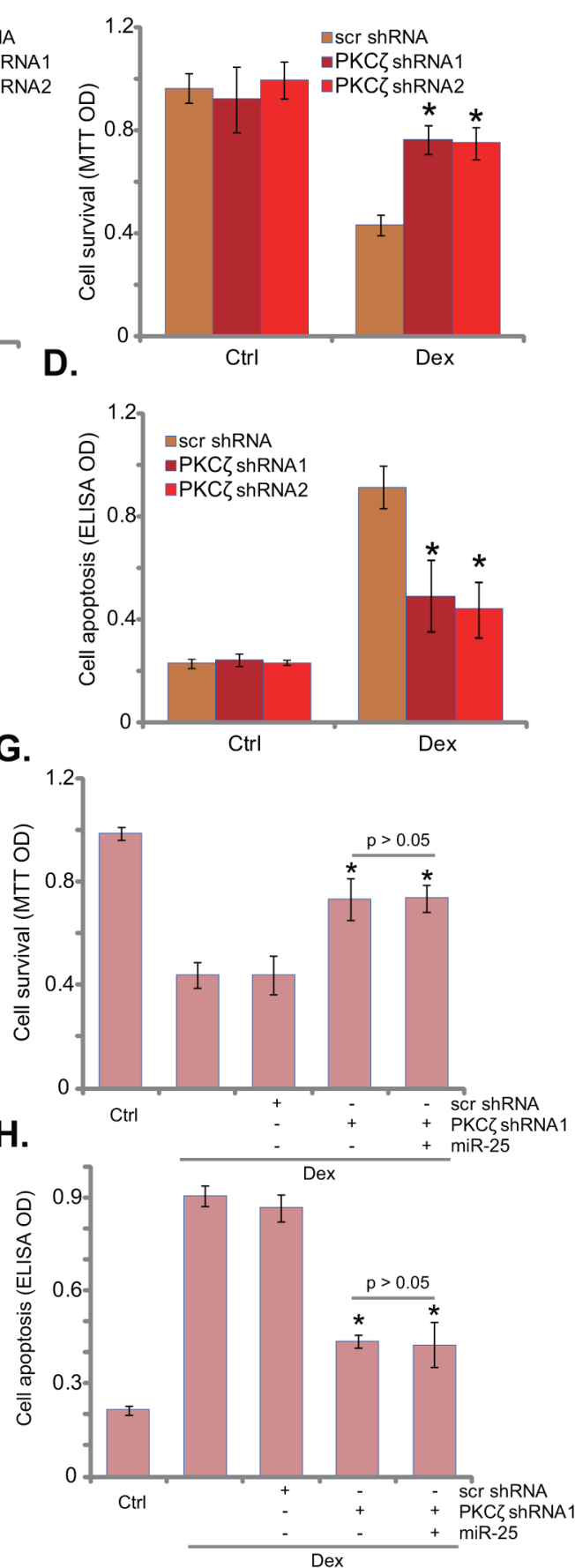

Figure 4: PKC $\zeta$ knockdown activates AMPK and protects human osteoblastic cells from Dex. OB-6 cells were infected with lentiviral PKC $\zeta$ shRNA ("-1 or -2") or non-sense control shRNA ("scr shRNA"), and stable cells were established; miR-25-5p and PKC $\zeta$ mRNA expression levels in these cells were tested A., PKC $\zeta$ protein expression and AMPK activation were also tested B. Above cells were treated with or without Dex $(1 \mu \mathrm{M})$ for 24 hours, cell viability $(\mathbf{C}$, MTT assay) and apoptosis (D, Histone DNA ELISA assay)

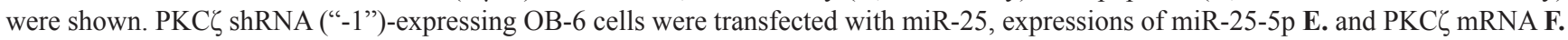
were shown; These cells were also treated with or without Dex $(1 \mu \mathrm{M})$ for 24 hours, cell viability G. and apoptosis H. were tested. "Ctrl" stands for untreated control group. Experiments in this figure were repeated three times, and similar results were obtained. * $p<0.05$ vs. "scr shRNA" cells (A, C-H). 
miR-25-induced AMPK activation was almost blocked by AMPK $\alpha$ shRNA or mutation. Importantly, miR-25induced cytoprotection against Dex was also abolished in AMPK $\alpha$-silenced or -mutated cells (Figure 5B and 5C). Therefore, miR-25 was no longer cytoprotective when AMPK was silenced or mutated (Figure $5 \mathrm{~B}$ and $5 \mathrm{C}$ ). It should be noted that AMPK $\alpha$ shRNA or mutation augmented Dex-induced damages in OB-6 cells (no miR25 , Figure $5 \mathrm{~A}-5 \mathrm{C}$ ). These results are consistent with the antigomir-25 results (Figure 3), and further confirmed that AMPK inhibition would potentiate Dex-induced cytotoxicity in osteoblastic cells. Therefore, AMPK activation is required for miR-25-induced cytoprotection in osteoblastic cells.

Existing evidences have shown that AMPK activation could inhibit Dex-induced ROS production, therefore protecting osteoblasts/osteoblastic cells [7]. ROS production was also observed in Dex-treated OB-6 cells (Figure 5D). Significantly, miR-25 expression decreased Dex-induced ROS production in OB-6 cells. Remarkably, such anti-oxidant function by miR-25 was almost blocked with AMPK silence or mutation (Figure 5D). Since AMPK activation may exert anti-oxidant function via activating NADPH [7, 18, 23, 25]. The NADPH activity was then tested. Indeed, miR-25 increased NADPH activity in OB-6 cells (Figure 5E). AMPK shRNA knockdown or dominant negative mutation almost nullified miR-25-induced NADPH activation (Figure 5E). Based on the above results, we propose that miR-25 expression activates AMPK signaling to inhibit oxidative stress, and eventually protects osteoblastic cells from Dex.

\section{miR-25-5p upregulation correlates with $\mathrm{PKC} \zeta$ downregulation and AMPK activation in human osteonecrosis tissues}

It has been shown that AMPK activation was upregulated in patients' necrotic femoral head tissues [23]. Here, miR-25-5p expression level in human necrotic femoral head tissues was tested, and its level was compared with that in the surrounding normal femoral head tissues (Figure 6A). In the necrotic tissues, the level of miR-25-5p was upregulated (Figure 6A), yet PKC $\zeta$ (protein and mRNA) level was decreased (Figure 6B-6D). AMPK activity, indicated by $\mathrm{p}$-ACC, was also increased in the necrotic femoral head tissues (Figure 6C). Therefore, miR-25 upregulation and $\mathrm{PKC} \zeta$ depletion could be at least one reason of AMPK activation in patients' necrotic femoral head tissues [23].

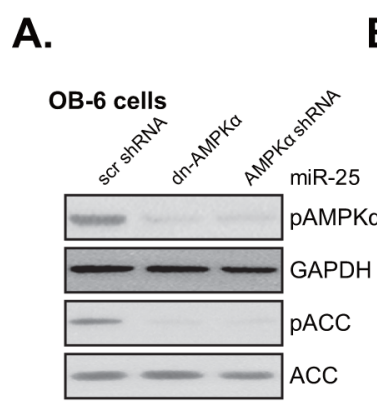

B.

C.
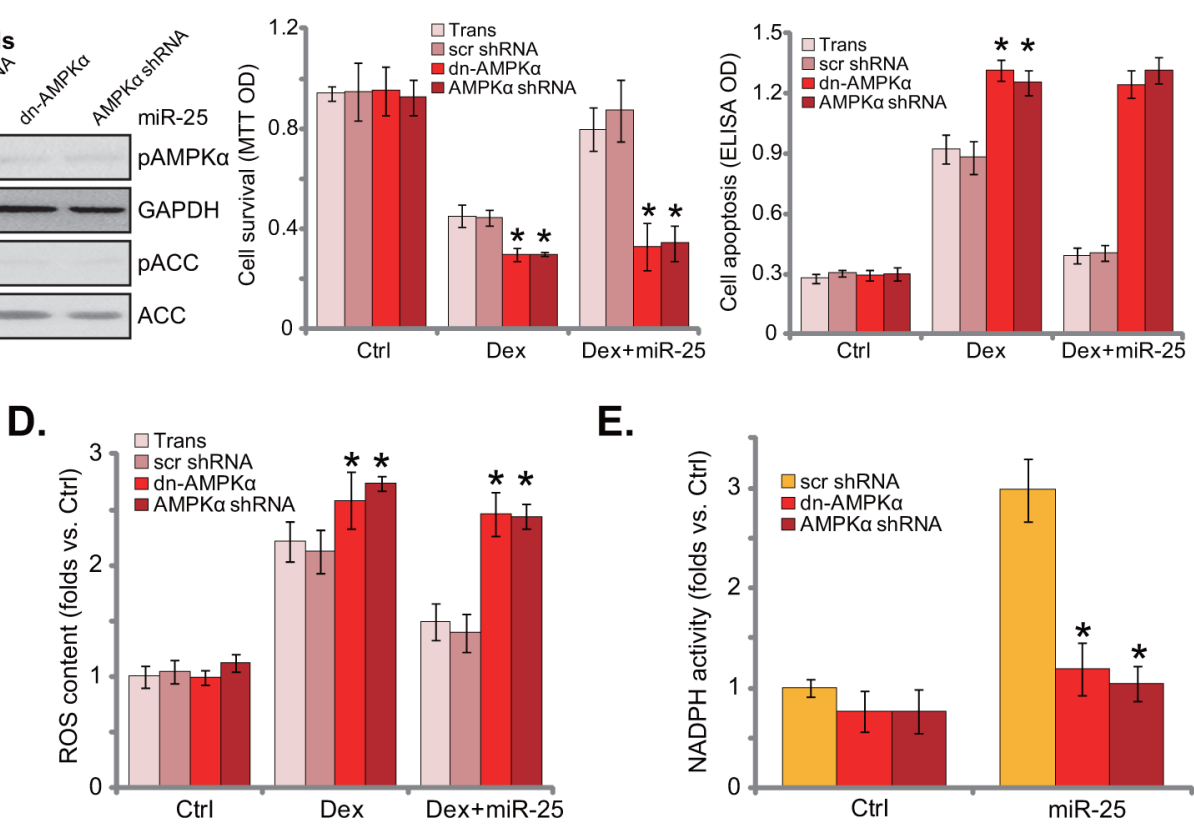

E.

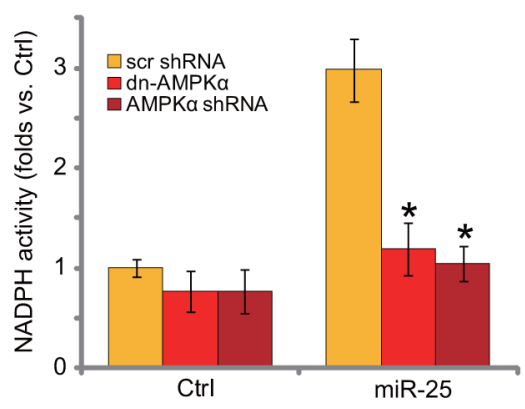

Figure 5: AMPK activation is required for miR-25-induced cytoprotection in osteoblastic cells. Stable OB-6 cells with miR-25 were constructed with dominant negative AMPK $\alpha$ ("dn-AMPK $\alpha$ ", T172A), AMPK $\alpha$ shRNA, or the scramble control shRNA ("scr shRNA"), expressions of listed proteins in these cells were tested by Western blots A. Above cells were treated with or without Dex (1 $\mu \mathrm{M}$ ), cell viability (MTT assay, 24 hours, B., apoptosis intensity (Histone DNA ELISA assay, 24 hours, C. and ROS content (DCFH-DA fluorescent dye assay, 6 hours, D. were tested; NADPH activity in above cells was also shown (4 hours, E). Experiments in this figure were repeated three times, and similar results were obtained. "Ctrl" stands for untreated control group. * $p<0.05$ vs. "scr shRNA" cells. 
A.

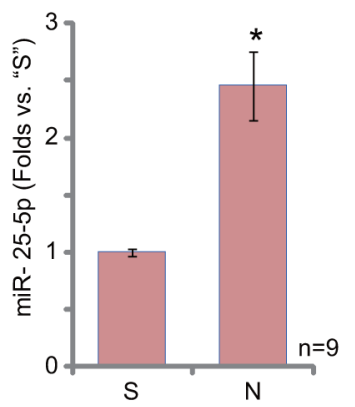

N. Necrotic femoral head tissues

S. Surrounding normal femoral head tissues
C.

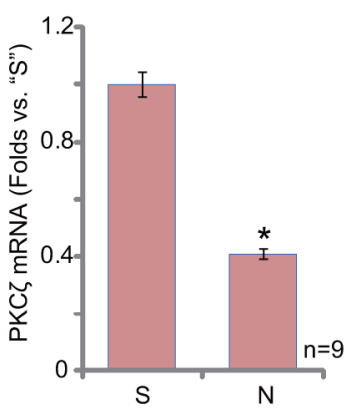

D.

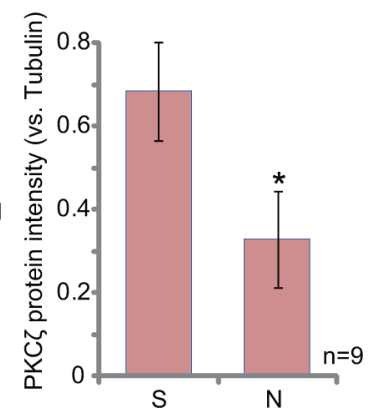

Figure 6: miR-25-5p upregulation correlates with PKC $\zeta$ downregulation and AMPK activation in human osteonecrosis tissues. Expressions of miR-25-5p A. and PKC $\zeta$ mRNA B. in surgery-isolated femoral head tissues (both normal or "S" and necrotic or "N") from GC-taking patients (n=9) were tested by qRT-PCR assay; Expression of listed protein was tested by Western blots $(\mathbf{C}$, and PKC $\zeta$ expression was quantified in $\mathbf{D})$. * $p<0.05$ vs. "S" tissues.

\section{DISCUSSION}

miR-25 is a member of miR-106b 25 cluster, which includes miR-106b, miR-93 and miR-25 [26]. In many cancers, miR-25 acts as an oncogene, and exerts pro-growth, anti-apoptotic, cell cycle-promoting activity via regulation of its target genes, including Bim, p57 and death receptor 4 (DR4) [27-29]. Its role in osteoblasts/ osteoblastic cells has not been extensively studied. Here, miR-25 expression is pro-survival in human osteoblastic cells. Expression of miR-25 inhibited Dexinduced osteoblastic cell death and apoptosis. Reversely, antagomiR-25 intensified osteoblastic cell death by Dex.

Although miR-25-5p is a predicted target of $\mathrm{PKC} \zeta$, it appears to be not conserved. As a matter of fact, the miRNA database (TargetScan v7.1) fails to find a single conserved miRNA for PKC . This could be due to the fact that the entire 3'UTR of $\mathrm{PKC} \zeta$ is poorly conserved among different cell types. Fortunately, here we show that miR25-5p targets and downregulates PKC $\zeta$ in osteoblastic cells, which activates AMPK signaling and protects cells against Dex. It will be interesting to test the potential effect of miR-25-5p on PKC $\zeta$ and AMPK signaling in other cells.

It has been shown that Dex kills osteoblasts/ osteoblastic cells via inducing oxidative stress $[7,30]$. Reversibly, ROS inhibition could protect osteoblasts/ osteoblastic cells from Dex [7]. Intriguingly, AMPK activation can exert anti-oxidant function and protect stressed cells [7, 18, 23, 31]. AMPK phosphorylates and inhibits ACC, thus reducing NADPH consumption [18]. Meanwhile, activated AMPK promotes NADPH synthesis though fatty-acid oxidation [18]. Here, miR-25 activated AMPK-NADPH signaling and inhibited Dex-induced ROS production. On the other hand, AMPK silence or mutation almost abolished miR-25-meidated anti-oxidant and cytoprotection in osteoblastic cells.

Excessive Dex usage could lead to osteoporosis $[1,2]$ and/or osteonecrosis [3]. In the Dex-taking patients' bones, decreased number of osteoblasts and increased number of apoptotic osteoblast cells are often detected $[1,2]$. Here, the in vitro results showed that miR-25-5p downregulated $\mathrm{PKC} \zeta$ and activated AMPK signaling to protect osteoblastic cell from Dex. Intriguingly, in human necrotic femoral head tissues, miR-25-5p expression was significantly increased, which was correlated with $\mathrm{PKC} \zeta$ downregulation and AMPK activation. Thus, in the following studies, it will be very interesting to further test the possible effect of miR-25-5p in animal model of Dex-induced bone damages.

In summary, these results conclude that miR-25 targets $\mathrm{PKC} \zeta$ and protects osteoblastic cells from Dex via activating AMPK signaling.

\section{MATERIALS AND METHODS}

\section{Chemicals and reagents}

Dex was obtained from Sigma Aldrich (Shanghai, China). All cell culture reagents were obtained from Gibco (Shanghai, China). All the antibodies were purchased from Santa Cruz Biotech (Santa Cruz, CA).

\section{Cell culture}

The OB-6 [4] and hFOB1.19 [32] human osteoblastic cells were obtained from the Cell Bank of Shanghai Institute of Biological Science (Shanghai, China). Osteoblastic cells were cultured as described $[4,32]$. 


\section{Quantitative real-time polymerase chain reaction (qRT-PCR) assay}

As described in our previous studies [9, 11], total RNA was extracted by the SV total RNA purification system (Promega, Shanghai, China). Extracted RNA was reverse-transcribed through the reverse transcriptase (Promega). cDNA derived from $500 \mathrm{ng}$ of RNA was amplified by quantitative real-time polymerase chain reaction ("qRT-PCR"). The SYBR Green PCR kit (Applied Biosystems, San Diego, CA) was applied to detect expression of targeted mRNAs. GAPDH primers (F-5'-AAG GTG AAG GTC GGA GTC-3' and R-5'-TGT AGT TGA GGT CAA TGA AGG-3') and $P K C \zeta$ primers (F-5'-GCG TAC TGC GGC CAG TGC-3' and R-5'-CTT GGC ATA GCT TCC ACG-3') were described [33]. PCR was performed in triplicate and was conducted using a Real-Time PCR Detection System (7500; ABI, Shanghai, China). mRNA expression was quantified using the ${ }^{\Delta \Delta} \mathrm{Ct}$ method using $G A P D H$ as the internal control. Mature hsa-miR-25-5p expression was assessed using TaqMan microRNA assay using the primer described [34]. Mature hsa-miR-25-3p primer was described previously [35]. Five ng of total RNA was reverse-transcribed using TaqMan MicroRNA Reverse Transcription kit (Applied Biosystem) and the looped primer provided by the specific TaqMan microRNA assay [36]. All the primers and sequences were synthesized by Genepharm (Shanghai, China).

\section{miR-25/antagomiR-25 expression}

Pre-miR-25, purchased from Applied Biosystem, was sub-cloned into pSuper-neo (OligoEngine, Seattle, WA) to generate miR-25 expression vector, which was transfected to the osteoblastic cells via Lipofectamine 2000 protocol (Invitrogen, Shanghai, China). Afterwards, cells were subjected to neomycin $(1.0 \mu \mathrm{g} / \mathrm{mL})$ selection for 10-12 days. Control cells were transfected with nonsense scramble microRNA-control ("miR-C") (a gift from Dr. Lu's group [37]). The antagomiR-25 expression vector was described previously [38] and was transfected to osteoblastic cells with Lipofectamine 2000. Stable cells were established via selection. Mature miR-25 expression in the stable cells was tested by the qRT-PCR assay.

\section{Western blot assay}

As described $[9,11]$, cell lysates were extracted via RIPA lysis buffer (Bio-sky, Nanjing, China). Aliquots of $30 \mu \mathrm{g}$ lysates per sample were electro-transferred on $10 \%$ SDS-PAGE gel, following by transfer to PVDF membranes. The blots were then incubated with designated primary and secondary antibodies. The antigen-antibody binding was detected via enhanced chemiluminescence (ECL) reagents. ImageJ software was applied to quantify protein band.

\section{Cell death detection}

Cell death was tested by counting cells using a cytometer after addition of trypan blue, which stained the cytoplasm of dead cells. Cell death percentage $(\%)=$ the number of trypan blue stained cells/the number of total cells $(\times 100 \%)[11]$.

\section{Cell viability assay}

Cell viability was measured via the routine 3-[4,5-dimethylthylthiazol-2-yl]-2,5 diphenyltetrazolium bromide (MTT) assay described in our previous studies $[9,11]$.

\section{Apoptosis assay by enzyme-linked immunosorbent assay (ELISA)}

As described [39], the Histone-DNA Apoptosis ELISA Detection Kit (Roche, Palo Alto, CA) was applied to quantify cell apoptosis with indicated treatment.

\section{shRNA knock and stable cell selection}

The two lentiviral shRNAs (GV248-puromycin vector) against human $\mathrm{PKC} \zeta$ were designed, synthesized and verified by Genepharm Co. (Shanghai, China). The AMPK $\alpha$ shRNA was described in our previous study [39]. Osteoblastic cells were seeded onto 6-well plates with $50 \%$ of confluence. The lentiviral shRNA $(10 \mu \mathrm{L} /$ $\mathrm{mL}$ ) were added to cultured cells for 24 hours. Afterwards, cells were cultured in puromycin $(1 \mu \mathrm{g} / \mathrm{mL})$-containing complete medium, until resistant colonies can be identified (10-14 days). The expression of target protein (PKC $\zeta$ or AMPK $\alpha$ ) in the stable cells was tested by Western blot assay. The scramble lentiviral shRNA (Santa Cruz) was added to the control osteoblastic cells.

\section{AMPK dominant negative mutation}

The dominant-negative mutant of AMPK $\alpha$ (dn-AMPK $\alpha$, T172A) construct was a gift from Dr. Lu's group [40], which was transfected to osteoblastic cells via Lipofectamine 2000 [40], and stable cells were selected via neomycin $(1 \mu \mathrm{g} / \mathrm{mL}$, Sigma). Transfection efficiency was always verified via Western blot assay in the stable cells.

\section{NADPH activity assay}

NADPH activity assay was described in previous studies [23, 41]. Briefly, after treatment of cells, the lysates were incubated with NADP-cycling buffer plus glucose-6-phosphate dehydrogenase (G6PD, Sigma) at $60^{\circ} \mathrm{C}$ for $30 \mathrm{~min}$ [23]. Afterwards, glucose 6-phosphate (G6P, Sigma) was added to the mixture, and the change in absorbance at $570 \mathrm{~nm}$ was measured every $30 \mathrm{~s}$ 
for $4 \mathrm{~min}$ at $30^{\circ} \mathrm{C}$. The concentration of NADP+ was calculated by subtracting [NADPH] from [total NADP]. NADPH activity was then calculated through NADPH/ $\mathrm{NADP}+[41]$.

\section{Reactive oxygen species (ROS) assay}

ROS production was measured via a DCFH-DA fluorescent dye (Invitrogen). After treatment, cells were incubated with $1 \mu \mathrm{M}$ of DCFH-DA (Invitrogen) for 30 min. Cells were then washed and analyzed for fluorescence using the flow cytometer (BD, Shanghai, China). The ROS intensity in the treatment group was normalized to that of control group.

\section{Human tissue specimens}

Surgery-isolated fresh necrotic femoral head tissues and their surrounding normal femoral head tissues were collected from Dex-taking patients. Fresh tissue specimens were dissolved in tissue lysis buffer (BiYunTian Biotechnology Research Institute, Nantong, China) and were subjected to qRT-PCR assay and Western blot assay. The experiment protocols requiring human samples were approved by the institutional review board and ethics committee of all authors' institutions, and written informed consent was obtained from each patient. A total of 9 patients were included. All studies were conducted according to the principles expressed in the Declaration of Helsinki.

\section{Statistics}

The data presented were mean \pm standard deviation (SD). Statistical differences were analyzed by one-way ANOVA followed by multiple comparisons performed with post hoc Bonferroni test (SPSS version 18.0). Values of $p<0.05$ were considered statistically significant.

\section{ACKNOWLEDGMENTS}

This work was generously supported by grants from the National Natural Science Foundation of China (81501866), and the Science and Technology Program of Nantong (MS22015051).

\section{CONFLICTS OF INTEREST}

The authors have no conflict of interests.

\section{Author contributions}

All authors carried out the experiments, participated in the design of the study and performed the statistical analysis, conceived of the study, and participated in its design and coordination and helped to draft the manuscript. All authors read and approved the final manuscript.

\section{REFERENCES}

1. den Uyl D, Bultink IE, Lems WF. Advances in glucocorticoid-induced osteoporosis. Curr Rheumatol Rep. 2011; 13: 233-40.

2. Weinstein RS. Clinical practice. Glucocorticoid-induced bone disease. N Engl J Med. 2011; 365: 62-70.

3. Kerachian MA, Seguin C, Harvey EJ. Glucocorticoids in osteonecrosis of the femoral head: a new understanding of the mechanisms of action. J Steroid Biochem Mol Biol. 2009; 114: 121-8.

4. Ding H, Wang T, Xu D, Cha B, Liu J, Li Y. Dexamethasoneinduced apoptosis of osteocytic and osteoblastic cells is mediated by TAK1 activation. Biochem Biophys Res Commun. 2015; 460: 157-63.

5. Yun SI, Yoon HY, Jeong SY, Chung YS. Glucocorticoid induces apoptosis of osteoblast cells through the activation of glycogen synthase kinase 3beta. J Bone Miner Metab. 2009; 27: 140-8.

6. Guo S, Xie Y, Fan JB, Ji F, Wang S, Fei H. alpha-Melanocyte stimulating hormone attenuates dexamethasone-induced osteoblast damages through activating melanocortin receptor 4-SphK1 signaling. Biochem Biophys Res Commun. 2016; 469: 281-7.

7. Guo S, Mao L, Ji F, Wang S, Xie Y, Fei H, Wang XD. Activating AMP-activated protein kinase by an alphal selective activator Compound 13 attenuates dexamethasoneinduced osteoblast cell death. Biochem Biophys Res Commun. 2016.

8. Ji F, Mao L, Liu Y, Cao X, Xie Y, Wang S, Fei H. K6PC-5, a novel sphingosine kinase 1 (SphK1) activator, alleviates dexamethasone-induced damages to osteoblasts through activating SphK1-Akt signaling. Biochem Biophys Res Commun. 2015; 458: 568-75.

9. Fan JB, Liu W, Zhu XH, Yuan K, Xu DW, Chen JJ, Cui ZM. EGFR-AKT-mTOR activation mediates epiregulininduced pleiotropic functions in cultured osteoblasts. Mol Cell Biochem. 2015; 398: 105-13.

10. Zhen YF, Wang GD, Zhu LQ, Tan SP, Zhang FY, Zhou XZ, Wang XD. P53 dependent mitochondrial permeability transition pore opening is required for dexamethasoneinduced death of osteoblasts. J Cell Physiol. 2014; 229: 1475-83.

11. Fan JB, Liu W, Yuan K, Zhu XH, Xu DW, Chen JJ, Cui ZM. EGFR trans-activation mediates pleiotrophin-induced activation of Akt and Erk in cultured osteoblasts. Biochem Biophys Res Commun. 2014; 447: 425-30.

12. Plotkin LI, Weinstein RS, Parfitt AM, Roberson PK, Manolagas SC, Bellido T. Prevention of osteocyte and osteoblast apoptosis by bisphosphonates and calcitonin. J Clin Invest. 1999; 104: 1363-74. 
13. Gohel A, McCarthy MB, Gronowicz G. Estrogen prevents glucocorticoid-induced apoptosis in osteoblasts in vivo and in vitro. Endocrinology. 1999; 140: 5339-47.

14. O'Neill LA, Hardie DG. Metabolism of inflammation limited by AMPK and pseudo-starvation. Nature. 2013; 493: 346-55.

15. Hardie DG, Ross FA, Hawley SA. AMPK: a nutrient and energy sensor that maintains energy homeostasis. Nat Rev Mol Cell Biol. 2012; 13: 251-62.

16. Hardie DG. AMP-activated/SNF1 protein kinases: conserved guardians of cellular energy. Nat Rev Mol Cell Biol. 2007; 8: 774-85.

17. Wang S, Song P, Zou MH. AMP-activated protein kinase, stress responses and cardiovascular diseases. Clin Sci. 2012; 122: 555-73.

18. Jeon SM, Chandel NS, Hay N. AMPK regulates NADPH homeostasis to promote tumour cell survival during energy stress. Nature. 2012; 485: 661-5.

19. Kim I, He YY. Targeting the AMP-Activated Protein Kinase for Cancer Prevention and Therapy. Front Oncol. 2013; 3: 175.

20. Mihaylova MM, Shaw RJ. The AMPK signalling pathway coordinates cell growth, autophagy and metabolism. Nat Cell Biol. 2011; 13: 1016-23.

21. Hunter RW, Foretz M, Bultot L, Fullerton MD, Deak M, Ross FA, Hawley SA, Shpiro N, Viollet B, Barron D, Kemp BE, Steinberg GR, Hardie DG, et al. Mechanism of action of compound-13: an alpha1-selective small molecule activator of AMPK. Chem Biol. 2014; 21: 866-79.

22. Zhu Y, Zhou J, Ao R, Yu B. A-769662 protects osteoblasts from hydrogen dioxide-induced apoptosis through activating of AMP-activated protein kinase (AMPK). Int $\mathrm{J}$ Mol Sci. 2014; 15: 11190-203.

23. She C, Zhu LQ, Zhen YF, Wang XD, Dong QR. Activation of AMPK protects against hydrogen peroxide-induced osteoblast apoptosis through autophagy induction and NADPH maintenance: New implications for osteonecrosis treatment? Cell Signal. 2014; 26: 1-8.

24. Deepa SS, Zhou L, Ryu J, Wang C, Mao X, Li C, Zhang N, Musi N, DeFronzo RA, Liu F, Dong LQ. APPL1 mediates adiponectin-induced LKB1 cytosolic localization through the PP2A-PKCzeta signaling pathway. Mol Endocrinol. 2011; 25: 1773-85.

25. Balteau M, Van Steenbergen A, Timmermans AD, Dessy C, Behets-Wydemans G, Tajeddine N, Castanares-Zapatero D, Gilon P, Vanoverschelde JL, Horman S, Hue L, Bertrand L, Beauloye C. AMPK activation by glucagon-like peptide-1 prevents NADPH oxidase activation induced by hyperglycemia in adult cardiomyocytes. Am J Physiol Heart Circ Physiol. 2014.

26. Petrocca F, Vecchione A, Croce CM. Emerging role of miR106b-25/miR-17-92 clusters in the control of transforming growth factor beta signaling. Cancer Res. 2008; 68: 8191-4.
27. Zhang H, Zuo Z, Lu X, Wang L, Wang H, Zhu Z. MiR25 regulates apoptosis by targeting Bim in human ovarian cancer. Oncol Rep. 2012; 27: 594-8.

28. Razumilava N, Bronk SF, Smoot RL, Fingas CD, Werneburg NW, Roberts LR, Mott JL. miR-25 targets TNFrelated apoptosis inducing ligand (TRAIL) death receptor-4 and promotes apoptosis resistance in cholangiocarcinoma. Hepatology. 2012; 55: 465-75.

29. Wu C, Li M, Hu C, Duan H. Clinical significance of serum miR-223, miR-25 and miR-375 in patients with esophageal squamous cell carcinoma. Mol Biol Rep. 2014; 41: 1257-66.

30. Almeida M, Han L, Ambrogini E, Weinstein RS, Manolagas SC. Glucocorticoids and tumor necrosis factor alpha increase oxidative stress and suppress Wnt protein signaling in osteoblasts. J Biol Chem. 2011; 286: 44326-35.

31. Zheng K, Lu H, Sheng Z, Li Y, Xu B. Low-concentration of perifosine surprisingly protects cardiomyocytes from oxygen glucose deprivation. Biochem Biophys Res Commun. 2016; 469: 753-60.

32. Xu Y, Li G, Du B, Zhang P, Xiao L, Sirois P, Li K. Hepcidin increases intracellular $\mathrm{Ca} 2+$ of osteoblast hFOB1.19 through L-type Ca2+ channels. Regul Pept. 2011; 172: 58-61.

33. Lin CC, Lin CE, Lin YC, Ju TK, Huang YL, Lee MS, Chen JH, Lee H. Lysophosphatidic acid induces reactive oxygen species generation by activating protein kinase $\mathrm{C}$ in PC-3 human prostate cancer cells. Biochem Biophys Res Commun. 2013; 440: 564-9.

34. Gao W, Chan JY, Wong TS. Curcumin exerts inhibitory effects on undifferentiated nasopharyngeal carcinoma by inhibiting the expression of miR-125a-5p. Clin Sci. 2014; 127: 571-9.

35. Li M, Song Q, Li H, Lou Y, Wang L. Circulating miR25-3p and miR-451a May Be Potential Biomarkers for the Diagnosis of Papillary Thyroid Carcinoma. PLoS One. 2015; 10: e0132403.

36. Marchi S, Lupini L, Patergnani S, Rimessi A, Missiroli S, Bonora M, Bononi A, Corra F, Giorgi C, De Marchi E, Poletti F, Gafa R, Lanza G, et al. Downregulation of the mitochondrial calcium uniporter by cancer-related miR-25. Curr Biol. 2013; 23: 58-63.

37. Chen MB, Wei MX, Han JY, Wu XY, Li C, Wang J, Shen W, Lu PH. MicroRNA-451 regulates AMPK/mTORC1 signaling and fascin 1 expression in HT-29 colorectal cancer. Cell Signal. 2014; 26: 102-9.

38. Li BS, Zuo QF, Zhao YL, Xiao B, Zhuang Y, Mao XH, Wu C, Yang SM, Zeng H, Zou QM, Guo G. MicroRNA-25 promotes gastric cancer migration, invasion and proliferation by directly targeting transducer of ERBB2, 1 and correlates with poor survival. Oncogene. 2015; 34: 2556-65.

39. Zhu LQ, Zhen YF, Zhang Y, Guo ZX, Dai J, Wang $\mathrm{XD}$. Salinomycin activates AMP-activated protein 
kinase-dependent autophagy in cultured osteoblastoma cells: a negative regulator against cell apoptosis. PLoS One. 2013; 8: e84175.

40. Lu PH, Chen MB, Ji C, Li WT, Wei MX, Wu MH. Aqueous Oldenlandia diffusa extracts inhibits colorectal cancer cells via activating AMP-activated protein kinase signalings. Oncotarget. 2016; 7:4588945900. doi: 10.18632/oncotarget.9969.

41. Hu X, Jiang F, Bao Q, Qian H, Fang Q, Shao Z. Compound 13, an alpha1-selective small molecule activator of AMPK, potently inhibits melanoma cell proliferation. Tumour Biol. 2015. 